\title{
Chronic Inflammation and Malignancy in Ulcerative Colitis
}

\author{
Sai Sunkara, ${ }^{1}$ Garth Swanson, ${ }^{2}$ Christopher B. Forsyth, ${ }^{2}$ and Ali Keshavarzian ${ }^{2}$ \\ ${ }^{1}$ Department of Medicine, Rush-Presbyterian-St. Luke's Medical Center, Rush University, Chicago, IL 60612, USA \\ ${ }^{2}$ Section of Gastroenterology and Nutrition, Rush University Medical Center, 1745 West Harrison Street, \\ Professional Building, Suite 206, Chicago, IL 60612, USA \\ Correspondence should be addressed to Garth Swanson, garth_swanson@rush.edu
}

Received 12 December 2010; Accepted 1 February 2011

Academic Editor: T. Arakawa

Copyright () 2011 Sai Sunkara et al. This is an open access article distributed under the Creative Commons Attribution License, which permits unrestricted use, distribution, and reproduction in any medium, provided the original work is properly cited.

Ulcerative colitis (UC) is a chronic inflammatory bowel disease (IBD) associated with multiple colonic and extraintestinal complications, the most severe being the development of colorectal cancer (CRC). Compared to the general population, there is an increased risk of CRC associated with UC. Although the pathogenesis of CRC in UC is unknown, most studies have linked it to long-standing inflammation as well as other risk factors such as duration of disease, extent of inflammation, family history of CRC, and coexisting conditions such as primary sclerosing cholangitis (PSC). UC is a life-long disease for which patients enter a vigilant screening program which includes surveillance colonoscopy to promote early detection of CRC yet some controversies exist regarding the cost effectiveness of surveillance colonoscopy and improving survival. Newer modalities such as chromoendoscopy, narrow band imaging, high definition colonoscopy, and confocal microscopy have aided in developing a more targeted approach for early detection of dysplasia in surveillance colonoscopy. This review focuses on the role of chronic colonic inflammation and dysplasia in development of UC-associated CRC and current methods of screening, detection, chemoprevention, and treatment of UC-associated CRC.

\section{Introduction}

Ulcerative Colitis (UC) is one of two types of inflammatory bowel disease (IBD) with disease limited to the colonic mucosa. The disease is associated with episodic periods of debilitating symptoms such as abdominal pain, rectal bleeding, fecal urgency, diarrhea, incontinence, and weight loss followed by asymptomatic intervals of remission. UC also is associated with extraintestinal manifestations such as uveitis, ankylosing spondylitis, erythema nodosum, and primary sclerosing cholangitis (PSC). The disease course in UC may be severe, but main contributors to morbidity and mortality is due to the increased risk of developing colorectal cancer (CRC) [1].

The incidence of CRC in the UC is approximately 4/1000 per person year duration with an average prevalence of 3.5\% [2]. Currently, UC ranks third only behind familial adenomatous polyposis (FAP) and hereditary nonpolyposis colorectal cancer (HNPCC) as a high-risk condition for CRC [3]. While the exact pathogenesis behind the development of colitis related CRC has not been identified, studies have shown it is most likely a result of chronic inflammation that leads to progressive dysplasia and eventually adenocarcinoma. This neoplastic process typically begins after a long duration of disease of 8 to 10 years and it can occur despite excellent control of most UC disease symptoms and even control of colonic inflammation by medications [4]. Compared to sporadic CRC which develops from the mucosa-adenoma-cancer sequence, the development of CRC in UC occurs mainly in flat areas of the colon due to dysplasia [5]. Dysplasia is defined as an "unequivocal neoplastic alteration of the intestinal epithelium that is restricted to the basement membrane", and the use of this term as a way of quantifying the severity of inflammation was adopted by Riddell et al. in 1983 [6]. The direct association between dysplasia and CRC has been documented in patients who were found to have dysplasia and underwent prophylactic proctocolectomy; of these individuals, $90 \%$ were found to have CRC [7]. Once low-grade dysplasia has been found, the progression rate to high-grade dysplasia typically is $50 \%$ over 5 years and the eventual progression of high grade dysplasia to cancer is $25-45 \%$ [5]. Besides the development of CRC 
from dysplasia in flat areas of the colon, adenocarcinoma can also occur in raised mucosa called "dysplasia-associated lesions or masses" (DALM). DALM lesions are elevated areas known to have an increased risk for the development of adenocarcinoma in UC [8] and should practically be considered malignant and be managed like a cancer. It should be noted that DALM lesions should be differentiated from sporadic adenomatous polyp that be definition contains dysplastic epithelial cells and yet is not malignant and should be managed like any other adenomatous polyp by snare polypectomy. However, several studies have demonstrated the difficulty of differentiating dysplasia in DALM from adenoma. Presence of dysplasia in flat mucosa surrounding the raised lesion indicates a DALM rather than a simple adenoma polyp, and thus sampling surrounding mucosa is crucial when managing a polypoid lesion in patients with UC. The duration of disease, extent of inflammation, family history, and concomitant PSC are all risk factors which have been proven to increase the risk of CRC in UC [8].

Due to the increased risk of CRC, surveillance colonoscopy has become the standard method to detect dysplasia in individuals with UC. There is also increased interest in chemoprevention with salicylates and urodeoxycholic acid to stop the development of dysplasia [9]. Finally, in individuals with focal dysplasia, total proctolectomy has been shown to decrease the risk of CRC. This review article will mainly focus on the increased risk, surveillance, and chemoprevention of CRC in patients with UC.

\section{Literature Review}

2.1. The Incidence and Prevalence of CRC in UC. The risk of CRC in UC was first discovered by Crohn and Rosenberg in 1925 [10]. Over the past two decades, studies have been conducted to quantify the magnitude and analyze this risk. In 2001, Eaden et al. conducted a meta-analysis study which stratified the incidence of CRC in UC [2]. This study was able to analyze the risk of developing cancer based on age, duration of colitis and extent of disease during the time of diagnosis. This study was the first to attempt to analyze patients based on predefined inclusion criteria and went further to analyze how various risk factors in UC contributed to increasing the risk of CRC. One hundred ninety four studies reporting the risk of CRC in IBD were reviewed and of these 116 which met inclusion criteria were extracted for the meta-analysis. Studies which evaluated patients with Crohn's disease and included incidence of cancer mortality are examples of those excluded from the analysis. In general, 54, 478 patients with UC and 1698 cases with CRC were considered, and the overall prevalence of CRC was determined to be $3.7 \%$ [2]. The study was able to determine that there was a dramatic increase in development of CRC in long-standing UC when compared to the general population which the Office of National Statistics reported as $0.06 \%$. Also those with extensive disease defined as pancolitis were shown to have an increased rate of CRC at $5.4 \%$. The cumulative incidence over 10,20 , and 30 years was also reported as $1.6 \%, 8.3 \%$ and $18.4 \%$, respectively indicating a direct increased risk of CRC with longer disease duration
[2]. Following this study, further population-based studies were conducted which reported lower risks of CRC. Palli et al. conducted a cohort study using UC patients diagnosed between 1978 and 1992 and reported an incidence rate of $0.13 \%$ [11]. A randomized cohort study in Manitoba, Canada of 2672 UC patients found an annual incidence of $0.2 \%$ [12]. These studies indicated that there was a dramatic increase in the risk of developing CRC in long-standing UC when compared to the general population, and those with extensive disease were at the highest risk [2]. These studies also showed that incidence and prevalence of UCassociated $\mathrm{CRC}$ varies in different population, and it appears that the incidence is much lower in community cohort compared to university hospital cohort suggesting that more severe cases of UC (typically referred to and managed in academic hospitals). Prior to these studies, there were many controversies regarding the significance of individuals with UC developing CRC. Patients from tertiary referral centers with severe disease and even early stages of malignancy tended to elevate the risk of CRC in UC to as high as $60 \%$ whereas population-based studies which included patients with less severe disease reported markedly lower rates of CRC [13]. Dysplasia, the marker used in detection of CRC in UC, has also been subject to sampling error as different patients have presented at different grades of dysplasia, and it is difficult to compare dysplasia detected by colonoscopy with those that have undergone proctocolectomy, making it difficult to calculate true relative risk [14]. Yet based on the existing published, it is clear that individuals with UC are clearly at higher risk of developing CRC.

2.2. Risk Factors for CRC in UC. CRC has been linked to several specific risk factors seen in the setting of chronic UC: the duration of colitis, extent of inflammation, family history, and concurrent PSC $[2,3,8,15,16]$. UC has a bimodal age of onset and a natural history of a relapsing disease course with frequent episodes of flare, therefore predisposing individuals to an intermittently chronic state of mucosal inflammation [17]. These repetitive cycles of inflammation have been associated with development of extensive dysplasia and eventual progression to malignancy. A retrospective data study in Alberta, Canada of UC patients who developed CRC showed a 5.4\% CRC incidence rate in patients with pancolitis [18]. The study analyzed patients diagnosed with UC before and after 40 years of age. The median duration from diagnosis of UC to develop CRC was 16 years. The study concluded that those with longer duration of disease were at increased risk of developing CRC [18]. The study also showed that individuals who developed UC later in life were more likely to have disease which progressed more rapidly [18]. The age of diagnosis also has been proposed to be an independent risk factor for CRC [13]; yet, there is insufficient data to support this theory when controlling for disease extent. The risk of cancer was, however, found to be directly related to the burden and extent of disease when active inflammation and pancolitis were evident in the cases with CRC. The relative risk of cancer was $4 \mathrm{X}$ that for left-sided colitis and 20X for those with extensive colitis [19]. A retrospective review was conducted 
at Mt. Sinai Hospital of 100 UC patients diagnosed with CRC [20]. The age of onset at UC, age at diagnosis of CRC, and the duration from onset of UC to diagnosis of CRC were documented. Based on the data, the mean age of onset of UC was 27 years, the main distribution was left sided colitis, and an increase risk of CRC was found to occur approximately one decade later at median age of 36 . In that series, the mean age of diagnosis of CRC was 47 and 58 for extensive colitis and left-sided colitis, respectively. Using regression analysis, the age at onset of UC was compared to the age at diagnosis of cancer and a significant correlation was found between both extensive colitis $(P<.0001)$ and left-sided colitis $(P<$ .005) [20]. The study further concluded that early age of onset was not an absolute risk factor independent of longer disease duration [20]. Gyde et al. conducted a study which further supported this data and showed that older patients with extensive disease still developed CRC at a significant rate compared to patients diagnosed at a young age [15]. Backwash ileitis, which is the extension of inflammation in UC into the terminal ileum, has been suggested to also increase the risk of CRC but solid data to support this theory has yet to be published [21].

2.3. Clinical Features of Colitis-Related CRC versus Sporadic Colorectal Cancer. CRC is typically classified in terms of four distinct etiologies: inherited genetic instability as seen with hereditary nonpolyposis CRC, mutations in the adenomatous polyposis coli (APC) gene as seen in FAP, inflammation based malignancy as seen in UC, and sporadic. Sporadic colorectal cancer (SCRC) typically occurs in normal colonic mucosa, through the mucosa-adenoma-carcinoma sequence is the result of chromosomal instability, microsatellite instability, and hypermethylation [3, 22]. However, unlike normal colonic mucosa where precancerous adenomas are the precursor to invasive carcinoma, UC-associated CRC is driven by the inflammation-dysplasia-carcinoma sequence [6]. The inflamed mucosa seen in UC is more prone to the development of dysplasia which includes the abnormal crypt architecture, reduction of epithelial crypts which become branched and distorted, and the development of enlarged hyper chromatic cell nuclei [23]. The rate of dysplasia development correlates with the degree of inflammation driven. Individuals with colitis-associated CRC are therefore seen at a younger age (mean age of 43) [2] and usually presented with more invasive cancer. In SCRC, dysplasia is usually contained to polypoid adenoma which undergoes mutations, whereas in UC-related CRC, dysplasia is usually multifocal and is located in flat more difficult to detect areas of the colon [23].

2.4. Inflammation-Associated Malignancy. UC has been shown to increase the risk of CRC, and the etiology has been attributed to chronic inflammation. The chronic inflammation in IBD often results in increased reepithelialization of cells and cell turnover in the colonic mucosa and leads to increased risk of errors in the cell cycle repair. Active inflammation in colorectal mucosa is characterized by a predominant neutrophillic infiltration with crypt abscesses and ulceration of the epithelium. Inactive inflammation is marked by predominance of lymphocytes. Oxidative stress, the body's internal defense mechanism to detoxify and repair damage, also has been linked to chronic inflammation and genomic instability that promotes progression of UC to cancer. Inflammatory cells in colitis generate oxygen radicals and nitrogen species. Neutrophils and macrophages also generate free radicals. These radicals tend to affect a large array of metabolic processes involved in cell repair, one of which is p53, the tumor suppressor protein. P53 mutations occur early in colitis and are usually detected when no dysplasia is histologically seen [24]. A high frequency of p53 mutations has been found in chronic UC patients with severe disease who were not diagnosed with cancer [24]. Due to the increased oxidative stress and repetitive cell repair cycles the risk of accumulating oncogenic mutations in UC is high and ultimately results in dysplasia and the neoplastic change results in dysplasia and eventually invasive CRC in UC [5].

2.5. Dysplasia. Colitis-related CRC differs from sporadic CRC in that lesions are multifocal, discrete, and flat making detection more difficult yet genetic abnormalities such as alterations in the $A P C, p 53, b c l-2$, and $\mathrm{K}$-ras genes, microsatellite instability, and aneuploidy are still present [23]. In addition, colitis-related CRC also follows the inflammation-dysplasia-carcinoma sequence as noted above. However, it is important to note that this sequence does not always occur in this progression and at times cancer can be detected without at preceding dysplasia. It is also useful to note that LGD can evolve to cancer without progression to HGD [25]. Dysplasia is a histological premalignant precursor defined as "unequivocal neoplasia of the epithelium confined to the basement membrane without invasion into the lamina propria" [6]. Dysplasia can present at sites of inflammation as raised or flat alterations in the mucosa. Flat dysplasia is difficult to identify endoscopically and is detected via random biopsy specimens. Advances in endoscopy have aimed to find more effective techniques in detecting flat dysplasia [24]. Dysplasia can also present in a raised mass like lesions commonly referred to as DALM or "dysplasia-associated lesions or masses" which when resectable appear to behave similarly to adenoma precursors seen in SCRC [26].

2.6. DALM Lesions. DALM lesions as opposed to adenoma like polyps are considered high-risk unresectable lesions in regards to malignancy CRC and discovery of a DALM lesion typically warrants colectomy. Bernstein et al. conducted a study of 1225 patients who underwent colonoscopic surveillance. In individuals with DALM lesions, colectomy reported CRC in $43 \%$ of patients and specifically in those with high-grade dysplasia in flat mucosa was carcinoma was revealed in $42 \%$ to $67 \%$ of the colectomy specimens [27]. If a DALM is endosopically removed and biopsied and the area surrounding the polyp is also biopsied and with no evidence of dysplasia is detected, the performance of a colectomy is not needed [27] and it can be treated with polypectomy.

2.7. Histopathological Subjectivity of Dysplasia. In 1983, Riddell et al. formulated the classification system for different 
TABLE 1: Reasons for failure of surveillance colonoscopy.

(i) Noncompliance of patients with UC to follow up with gastroenterologists

(ii) Inadequate collection of biopsy samples from surveillance colonoscopy

(iii) Inability of pathologist to properly diagnose degree of dysplasia

(iv) Disagreement amongst pathologists in regards to degree of dysplasia

(v) Development of cancer prior to the initiation of surveillance colonoscopy

degrees of dysplasia and divided dysplasia into five categories: negative, low-grade, indefinite, high, and invasive cancer [6]. The presence of no objective marker, however, has lead to variability and discrepancies amongst pathologists when evaluating disease and continues to remain problematic $[6,28]$. The current practice in cases where the degree of dysplasia is controversial, a second pathologist's evaluation of the specimen is vital when making a diagnosis. In addition, for the purposes of most studies, dysplasia has been divided in to high or low grade for the purposes of clinical decision making.

\section{Prevention of CRC in UC}

3.1. Primary Prevention and Surveillance Screening. In addition to diagnosing IBD and evaluating the extent of disease, the primary approach to detect precancerous lesions and prevent progression to CRC in UC is surveillance colonoscopy, that is, early detection and secondary prevention of CRC [29]. Early detection of CRC has been associated with good prognosis; however, the direct correlation with performing surveillance colonoscopy and decreasing mortality in UC is debatable [30]. Detection is difficult in colitis-related CRC as opposed to sporadic CRC in that dysplasia tends to be multifocal and flat. The American Cancer Society's has set specific guidelines on the proper technique for surveillance colonoscopy. The guidelines state that surveillance colonoscopy should be performed every 1-2 years, beginning 8 years after the diagnosis of pancolitis has been established and 12-15 years after the diagnosis of left-sided colitis [31]. Since the risk of malignancy increases with duration of disease, and extent of disease, more frequent screening has also been indicated in cases with severe disease [32]. For patients with pancolitis, a colonoscopy should be performed every 1-2 years during the 2nd decade of disease and every year during the $3 \mathrm{rd}$ decade of disease [31]. In addition to suspicious areas, two to four random, serial biopsy specimens should be taken every $10 \mathrm{~cm}$ of the entire colon [31] and a minimum number of 36 biopsies should be taken from the colon [32]. Lastly individuals with PSC who are at increased risk of cancer should have an annual colonoscopy, starting at the time of diagnosis $[32,33]$. Despite these recommendations for surveillance colonoscopy, the Cochrane database review in 2004 concluded that although there was no evidence supporting surveillance colonoscopy as directly prolonging survival in IBD, it did decrease the risk of mortality in IBD [33-35].

3.2. Surveillance Programs in UC. In an effort to promote patient compliance and promote the detection of CRC in UC, a surveillance plan should be established with patients when the original diagnosis of UC is made which allows for patients to develop a firm relationship with their gastroenterologist and creates ground work in case the discussion regarding colectomy versus vigilant screening is required in the future $[36,37]$ (Figure 1). Despite promotion of surveillance colonoscopy, many factors are essential for effectively detecting and diagnosing dysplasia. Table 1 indicates primary reasons for the failures which results in the surveillance process [38]. Initial screening colonoscopy should be performed in all UC patients 8-10 years after the diagnosis of UC has been established [32]. A surveillance plan would also help with the ultimate goal to decrease the risk of development of CRC in UC which entails decreasing the degree of inflammation seen in UC and ultimately maintain remission. This recommendation is also applied to patients with Crohn's colitis since there is no significant difference in the risk of CRC between UC and Crohn's colitis.

3.3. Advances in Endoscopic Detection of Dysplasia and Cancer. Despite being the current standard of surveillance, no studies have shown a reduction in the risk of developing or preventing CRC due to colonoscopic surveillance; however, early detection has been shown to have better prognosis $[37,39]$. The difficulty of detecting dysplasia due to subtle and macroscopically invisible lesions has promoted advancements in imaging for a more targeted approach [32, 40, 41]. Chromoendoscopy uses various dyes which are applied to the colonic mucosa to allow for better visualization and has been shown to be three times more helpful in detecting flat dysplasia than conventional colonoscopy [42]. Two stains, indigo carmine, and methylene blue are injected via an endoscopic catheter to coat the colonic mucosa and highlight subtle mucosal lesions, active inflammation, and dysplasia. This technique allows targeted biopsies and has been reported to better detect neoplastic changes in the colon and aid in earlier detection of CRC [43]. Another advantage of chromoendoscopy is it allows for more detailed observation of crypt architecture and pit patterns which help differentiating neoplastic lesions. Chromoendoscopy does take longer to perform, but with more experience and the increasing sensitivity of the biopsies, it still appears to be a surveillance method superior to standard colonoscopy and has been recently recommended for surveillance [32, 44, 45]. An alternative and less labor intensive approach focuses on better visualization of the mucosal vasculature is narrow-band imaging (NBI). In NBI, the mucosal surface is enhanced by applying an optical filter to the light used for illumination and aids in visualizing the microvasculature in 


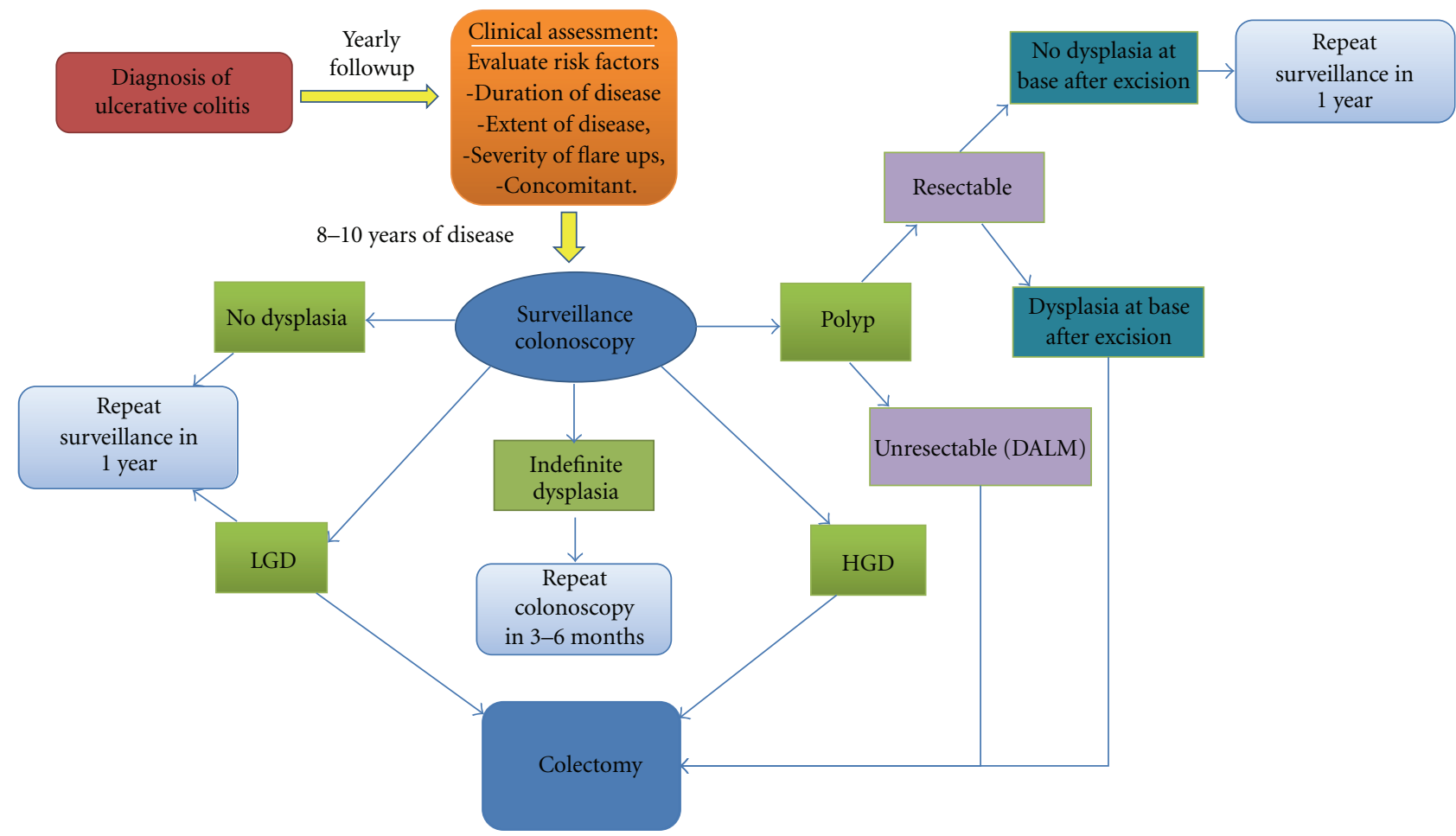

FIGURE 1: Example of a surveillance colonscopy algorithm based off guidelines proposed by the American Cancer Society [30].

addition to performing more target biopsies with the use of dye [46]. Dekker et al. conducted a randomized study of 42 patients with chronic UC who underwent NBI versus routine colonoscopy. At the conclusion of the study, 52 lesions were detected via NBI in 17 patients as opposed to only 28 in 13 patients who underwent colonoscopy [47]. Although NBI did increase the number of suspicious lesions detected, there is still insufficient data that it actually improves the detection of neoplasia to be applied to routine practice [48]. Confocal laser endomicroscopy, a technique used for detection of esophageal and gastric cancers has also gained publicity as it helps characterize suspicious lesions at a cellular and subcellular level by providing high-resolution images during ongoing endoscopy [49]. In confocal endomicroscopy, a microscope is built into the distal tip of the colonoscopy and essentially allows for histological evaluation of the mucosa as well as cellular and subcellular surfaces as the exam is performed. This method is very time consuming and, therefore, has been commonly combined with chromoendoscopy [50]. Studies have shown that combined techniques using chromoscopy and endomicroscopy have not only enabled for rapid diagnosis but have resulted in a 4.75 -fold increased detection of neoplastic lesions than conventional colonoscopy [51]. Further studies are needed to determine if endomicroscopy is a more effective surveillance method for the future.

3.4. Goals of Chemoprevention in UC. The current gold standard of secondary prevention of CRC in UC is via early detection by surveillance colonoscopy as this has shown to decrease overall mortality when detection of malignancy occurs early. However, surveillance colonoscopy is not always accurate with detection of dysplasia and furthermore does not stop the progression of colonic inflammation to dysplasia and carcinoma. Prophylactic colectomy currently is the most definitive approach to prevent CRC in high-risk patients with long-standing disease; however, many patients prefer to continued more frequent surveillance rather than undergoing an invasive surgery. An alternative and more desirable approach is primary prevention with chemopreventative agents; however, there is no universally accepted effective chemopreventative agent from CRC. Several potential chemopreventative agents have been suggested for inflammation-associated CRC. These include anti-inflammatory medications such as 5-ASA products (mesalamine and sulfasalazine) and nonsteroidal anti-inflammatory drugs (NSAIDs). Although there is still insufficient data to recommend use of these medications as chemopreventative agents on neoplastic progression, there is widespread use of these medications by patients and physicians due to the low side effect profile, availability, and the need to halting carcinogenesis and avoiding colectomy.

NSAIDs have been shown to decrease the risk of colon cancer by inhibiting the conversion of arachidonic acid to prostaglandins in the cyclo-oxygenase-2 pathway (COX-2) $[51,52]$. In a case controlled study by Bansal and Sonnenberg, patients with IBD and a comorbidity such as rheumatoid arthritis requiring NSAID usage were evaluated for CRC development. The study concluded that there was 
a significant reduction in mortality among those who used chronic NSAIDs [53]. Despite the potential benefits, the long-term usage of NSAIDs in chronic UC is still debated due to a high rate of side effects and toxicity, particularly the risk of NSAIS on the gastrointestinal tract for ulcers, and NSAIDs can also result in causing a disease flare $[54,55]$. Sulfasalazine also is another drug used in the treatment of mild to moderate colitis. It is split in the gut into sulfapyridine and 5-ASA, a derivative of acetylsalicylic acid (aspirin). Both sulfasalazine and 5-ASA reduce oxidative stress, inhibit cell proliferation, and promote apoptosis all of which are stages in the inflammation cascade. 5-ASA when used even in controlled disease not only provided protective effects but when used in a compliant individual, the risk of CRC was estimated to only $5 \%$ as opposed to $30 \%$ in a noncompliant patient $[56,57]$.

In patients with PSC and chronic UC, ursodeoxycholic acid (UDCA) in conjunction with 5-ASA has been shown to significantly decrease the risk of CRC. Pardi et al. conducted a randomized placebo controlled trial: 52 patients were followed for 355 person years on UDCA and showed a significant reduction risk of $74 \%$ for development of dysplasia or cancer [58]. Ursodeoxycholic acid is an antioxidant that decreases the concentration of the deoxycholic acid, a carcinogen in the colon and is the most effective chemopreventative drug proven to decrease the risk of CRC.

There is some compelling evidence to supports folate supplementation effect to decrease the prevalence of CRC $[59,60]$. Folate deficiency has been linked to the development of carcinogenesis and has been considered a potential risk factor for CRC. Active disease in UC results in decreased absorption and ultimately folate deficiency. Lashner et al. conducted two studies which ultimately supported folate supplementation as a chemoprotective method in preventing UC and found a direct correlation in regards to decreasing CRC rates in those given supplementation [61, 62]. The effects of dietary folate also were studied in IL-2 and beta 2-microglobulin-deficient mice with UC and subsequently developed CRC. The mice were divided into three dietary groups, and the study concluded that the incidence of highgrade dysplasia and adenocarcinoma in the group given supplemental folate was $46 \%$ lower than the group given no supplementation and that $4 \mathrm{X}$ the basal dietary supplementation of folate was required to halt the development of CRC [60].

Lastly immunomodulators such as 6-mercaptopurine or Azathiopurine are also used in UC; however, minimal data is available in regards to chemoprotective effects; these agents might be able to provide [63]. In summary, although more common in clinical practice due to wide availability, controversy still exists whether there is enough significant clinical evidence to recommend chemoprotective agents in all patients with UC; however, based on current findings the usage has been increasing promoted.

\section{Conclusion}

UC can be a debilitating disease which can lead to CRC secondary to chronic inflammation and the inflammationdysplasia-carcinoma sequence. Patients are recommended to undergo a cautious screening program with surveillance colonoscopy after 7-10 years of diagnosis. Dysplasia has been a helpful marker in the early detection of CRC in UC especially in endoscopic surveillance, and surveillance should be preformed with multiple random biopsies throughout the colon, and preferably with a method that allows for more targeted biopsies, such as chromoendoscopy. The use of chemopreventative agents, such as 5-ASAs or UDCA, are very appealing due to their availability and low side effect profile, and should be offered to high risk patients such as those with a family history or with PSC. While the cost effectiveness of surveillance colonoscopy is debatable, advancements in endoscopy are improving and should impact on the largest contributors of morbidity and mortality in colitis associated CRC.

\section{Abbreviations}

$\begin{array}{ll}\text { UC: } & \text { Ulcerative colitis } \\ \text { IBD: } & \text { Inflammatory bowel disease } \\ \text { CRC: } & \text { Colorectal cancer } \\ \text { PSC: } & \text { Primary sclerosing cholangitis } \\ \text { HNPCC: Hereditary nonpolyposis colorectal cancer } \\ \text { DALM: } & \text { Dysplasia associated lesions or masses } \\ \text { NSAIDs: } & \text { Nonsteroidal anti-inflammatory drugs } \\ \text { COX-2: } & \text { Cyclo-oxygenase-2 pathway } \\ \text { UDCA: } & \text { Ursodeoxycholic acid } \\ \text { NBI: } & \text { Narrow band imaging. }\end{array}$

\section{References}

[1] D. K. Podolsky, "Inflammatory bowel disease," The New England Journal of Medicine, vol. 347, no. 6, pp. 417-429, 2002.

[2] J. A. Eaden, K. R. Abrams, and J. F. Mayberry, "The risk of colorectal cancer in ulcerative colitis: a meta-analysis," Gut, vol. 48, no. 4, pp. 526-535, 2001.

[3] S. H. Itzkowitz and X. Yio, "Inflammation and cancer. IV. Colorectal cancer in inflammatory bowel disease: the role of inflammation," American Journal of Physiology, vol. 287, no. 1, pp. G7-G17, 2004.

[4] C. Mathy, K. Schneider, Y. Y. Chen, M. Varma, J. P. Terdiman, and U. Mahadevan, "Gross versus microscopic pancolitis and the occurrence of neoplasia in ulcerative colitis," Inflammatory Bowel Diseases, vol. 9, no. 6, pp. 351-355, 2003.

[5] M. N. Kulaylat and M. T. Dayton, "Ulcerative colitis and cancer," Journal of Surgical Oncology, vol. 101, no. 8, pp. 706$712,2010$.

[6] R. H. Riddell, H. Goldman, and D. F. Ransohoff, "Dysplasia in inflammatory bowel disease: standardized classification with provisional clinical applications," Human Pathology, vol. 14, no. 11, pp. 931-966, 1983.

[7] A. Von Herbay, C. Herfarth, and H. F. Otto, "Cancer and dysplasia in ulcerative colitis: a histologic study of 301 surgical specimen," Zeitschrift für Gastroenterologie, vol. 32, no. 7, pp. 382-388, 1994.

[8] J. Potack and S. H. Itzkowitz, "Colorectal cancer in inflammatory bowel disease," Gut and Liver, pp. 61-73, 2008.

[9] V. J. Croog, T. A. Ullman, and S. H. Itzkowitz, "Chemoprevention of colorectal cancer in ulcerative colitis," International Journal of Colorectal Disease, vol. 18, no. 5, pp. 392-400, 2003. 
[10] B. Crohn and H. Rosenberg, "The sigmoidoscopic picture of chronic ulcerative colitis (non-specific)," The American Journal of the Medical Sciences, vol. 170, pp. 220-228, 1925.

[11] D. Palli, G. Trallori, S. Bagnoli et al., "Hodgkin's disease risk is increased in patients with ulcerative colitis," Gastroenterology, vol. 119, no. 3, pp. 647-653, 2000.

[12] C. N. Bernstein, J. F. Blanchard, E. Kliewer, and A. Wajda, "Cancer risk in patients with inflammatory bowel disease: a population-based study," Cancer, vol. 91, no. 4, pp. 854-862, 2001.

[13] K. V. Winther, T. Jess, E. Langholz, P. Munkholm, and V. Binder, "Survival and cause-specific mortality in ulcerative colitis: follow-up of a population-based cohort in Copenhagen County," Gastroenterology, vol. 125, no. 6, pp. 1576-1582, 2003.

[14] J. Kewenter, H. Ahlman, and L. Hultén, "Cancer risk in extensive ulcerative colitis," Annals of Surgery, vol. 188, no. 6, pp. 824-828, 1978.

[15] S. N. Gyde, P. Prior, R. N. Allan et al., "Colorectal cancer in ulcerative colitis: a cohort study of primary referrals from three centres," Gut, vol. 29, no. 2, pp. 206-217, 1988.

[16] U. Broomé and A. Bergquist, "Primary sclerosing cholangitis, inflammatory bowel disease, and colon cancer," Seminars in Liver Disease, vol. 26, no. 1, pp. 31-41, 2006.

[17] E. Langholz, P. Munkholm, M. Davidsen, and V. Binder, "Course of ulcerative colitis: analysis of changes in disease activity over years," Gastroenterology, vol. 107, no. 1, pp. 3-11, 1994.

[18] C. J. Karvellas, R. N. Fedorak, J. Hanson, and C. K. W. Wong, "Increased risk of colorectal cancer in ulcerative colitis patients diagnosed after 40 years of age," Canadian Journal of Gastroenterology, vol. 21, no. 7, pp. 443-446, 2007.

[19] A. Sugita, D. B. Sachar, C. Bodian, M. B. Ribeiro, A. H. Aufses, and A. J. Greenstein, "Colorectal cancer in ulcerative colitis. Influence of anatomical extent and age at onset on colitiscancer interval," Gut, vol. 32, no. 2, pp. 167-169, 1991.

[20] A. Ekbom, C. Helmick, M. Zack, and H. O. Adami, "Ulcerative colitis and colorectal cancer: a population-based study," The New England Journal of Medicine, vol. 323, no. 18, pp. 12281233,1990

[21] U. A. Heuschen, U. Hinz, E. H. Allemeyer et al., "Backwash ileitis is strongly associated with colorectal carcinoma in ulcerative colitis," Gastroenterology, vol. 120, no. 4, pp. 841$847,2001$.

[22] J. P. J. Issa, N. Ahuja, M. Toyota, M. P. Bronner, and T. A. Brentnall, "Accelerated age-related CpG island methylation in ulcerative colitis," Cancer Research, vol. 61, no. 9, pp. 3573$3577,2001$.

[23] S. Itzkowitz, "Colon carcinogenesis in inflammatory bowel disease: applying molecular genetics to clinical practice," Journal of Clinical Gastroenterology, vol. 36, no. 5, pp. S70-S74, 2003.

[24] S. P. Hussain, P. Amstad, K. Raja et al., "Increased p53 mutation load in noncancerous colon tissue from ulcerative colitis: a cancer-prone chronic inflammatory disease," Cancer Research, vol. 60, no. 13, pp. 3333-3337, 2000.

[25] S. H. Itzkowitz, "Molecular biology of dysplasia and cancer in inflammatory bowel disease," Gastroenterology Clinics of North America, vol. 35, no. 3, pp. 553-571, 2006.

[26] M. O. Blackstone, R. H. Riddell, B. H. G. Rodgers, and B. Levin, "Dysplasia-associated lesion or mass (DALM) detected by colonoscopy in long-standing ulcerative colitis: an indication for colectomy," Gastroenterology, vol. 80, no. 2, pp. 366374, 1981
[27] C. N. Bernstein, F. Shanahan, and W. M. Weinstein, "Are we telling patients the truth about surveillance colonoscopy in ulcerative colitis?" The Lancet, vol. 343 , no. 8889, pp. 71-74, 1994.

[28] R. D. Odze, F. A. Farraye, J. L. Hecht, and J. L. Hornick, "Longterm follow-up after polypectomy treatment for adenoma-like dysplastic lesions in ulcerative colitis," Clinical Gastroenterology and Hepatology, vol. 2, no. 7, pp. 534-541, 2004.

[29] J. B. J. Fozard and M. F. Dixon, "Colonoscopic surveillance in ulcerative colitis-dysplasia through the looking glass," Gut, vol. 30, no. 3, pp. 285-292, 1989.

[30] J. Onkin, "Is colorectal cancer surveillance cost-effective in patients with ulcerative colitis?" Inflammatory Bowel Disease, vol. 14, pp. 196-197, 2008.

[31] T. Byers, B. Levin, D. Rothenberger, G. D. Dodd, and R. A. Smith, "American Cancer Society guidelines for screening and surveillance for early detection of colorectal polyps and cancer: update 1997," Ca: A Cancer Journal for Clinicians, vol. 47, no. 3, pp. 154-160, 1997.

[32] S. H. Itzkowitz, D. H. Present, V. Binder et al., "Consensus conference: colorectal cancer screening and surveillance in inflammatory bowel disease," Inflammatory Bowel Diseases, vol. 11, no. 3, pp. 314-321, 2005.

[33] R. C. Thomas, C. Selinger, and M. D. Rutter, "Adherence to BSG adenoma surveillance guidelines will reduce colonoscopic workload," Gut, vol. 54, no. 1, p. 173, 2005.

[34] T. L. Zisman and D. T. Rubin, "Colorectal cancer and dysplasia in inflammatory bowel disease," World Journal of Gastroenterology, vol. 14, no. 17, pp. 2662-2669, 2008.

[35] D. A. F. Lynch, A. J. Lobo, G. M. Sobala, M. F. Dixon, and A. T. R. Axon, "Failure of colonoscopic surveillance in ulcerative colitis," Gut, vol. 34, no. 8, pp. 1075-1080, 1993.

[36] M. J. Carter, A. J. Lobo, and S. P. L. Travis, "Guidelines for the management of inflammatory bowel disease in adults," Gut, vol. 53, no. 5, pp. v1-v16, 2004.

[37] A. Kornbluth and D. B. Sachar, "Ulcerative colitis practice guidelines in adults (update): American College of Gastroenterology, Practice Parameters Committee," American Journal of Gastroenterology, vol. 99, no. 7, pp. 1371-1385, 2004.

[38] T. Ullman, R. Odze, and F. A. Farraye, "Diagnosis and management of dysplasia in patients with ulcerative colitis and Crohn's disease of the colon," Inflammatory Bowel Diseases, vol. 15, no. 4, pp. 630-638, 2009.

[39] P. D. Collins, C. Mpofu, A. J. Watson, and J. M. Rhodes, "Strategies for detecting colon cancer and/or dysplasia in patients with inflammatory bowel disease," Cochrane Database of Systematic Reviews, no. 2, Article ID CD000279, 2006.

[40] G. N. J. Tytgat, V. Dhir, and N. Gopinath, "Endoscopic appearance of dysplasia and cancer in inflammatory bowel disease," European Journal of Cancer Part A, vol. 31, no. 7-8, pp. 1174-1177, 1995.

[41] M. Rutter, C. Bernstein, T. Matsumoto, R. Kiesslich, and M. Neurath, "Endoscopic appearance of dysplasia in ulcerative colitis and the role of staining," Endoscopy, vol. 36, no. 12, pp. 1109-1114, 2004

[42] R. Kiesslich, J. Fritsch, M. Holtmann et al., "Methylene blueaided chromoendoscopy for the detection of intraepithelial neoplasia and colon cancer in ulcerative colitis," Gastroenterology, vol. 124, no. 4, pp. 880-888, 2003.

[43] S. E. Kudo, S. Tamura, T. Nakajima, H. O. Yamano, H. Kusaka, and $\mathrm{H}$. Watanabe, "Diagnosis of colorectal tumorous lesions by magnifying endoscopy," Gastrointestinal Endoscopy, vol. 44, no. 1, pp. 8-14, 1996. 
[44] S. Winawer, R. Fletcher, D. Rex et al., "Colorectal cancer screening and surveillance: clinical guidelines and rationaleupdate based on new evidence," Gastroenterology, vol. 124, no. 2, pp. 544-560, 2003.

[45] D. P. Hurlstone, D. S. Sanders, A. J. Lobo, M. E. McAlindon, and S. S. Cross, "Indigo carmine-assisted high-magnification chromoscopic colonoscopy for the detection and characterisation of intraepithelial neoplasia in ulcerative colitis: a prospective evaluation," Endoscopy, vol. 37, no. 12, pp. 11861192, 2005.

[46] J. E. East, N. Suzuki, A. Von Herbay, and B. P. Saunders, "Narrow band imaging with magnification for dysplasia detection and pit pattern assessment in ulcerative colitis surveillance: a case with multiple dysplasia associated lesions or masses," Gut, vol. 55, no. 10, pp. 1432-1435, 2006.

[47] E. Dekker, F. J. C. van den Broek, J. B. Reitsma et al., "Narrowband imaging compared with conventional colonoscopy for the detection of dysplasia in patients with longstanding ulcerative colitis," Endoscopy, vol. 39, no. 3, pp. 216-221, 2007.

[48] K. Kuznetsov, R. Lambert, and J. F. Rey, "Narrow-band imaging: potential and limitations," Endoscopy, vol. 38, no. 1, pp. 76-81, 2006.

[49] M. Goetz, A. Hoffman, P. R. Galle, M. F. Neurath, and R. Kiesslich, "Confocal laser endoscopy: new approach to the early diagnosis of tumors of the esophagus and stomach," Future Oncology, vol. 2, no. 4, pp. 469-476, 2006.

[50] R. Kiesslich, M. Goetz, K. Lammersdorf et al., "Chromoscopyguided endomicroscopy increases the diagnostic yield of intraepithelial neoplasia in ulcerative colitis," Gastroenterology, vol. 132, no. 3, pp. 874-882, 2007.

[51] S. R. Ritland, J. A. Leighton, R. E. Hirsch, J. D. Morrow, A. L. Weaver, and S. J. Gendler, "Evaluation of 5-aminosalicylic acid (5-ASA) for cancer chemoprevention: lack of efficacy against nascent adenomatous polyps in the Apc(Min) mouse," Clinical Cancer Research, vol. 5, no. 4, pp. 855-863, 1999.

[52] R. N. DuBois, F. M. Giardiello, and W. E. Smalley, "Nonsteroidal antiinflammatory drugs, eicosanoids, and colorectal cancer prevention," Gastroenterology Clinics of North America, vol. 25, no. 4, pp. 773-791, 1996.

[53] P. Bansal and A. Sonnenberg, "Risk factors of colorectal cancer in inflammatory bowel disease," American Journal of Gastroenterology, vol. 91, no. 1, pp. 44-48, 1996.

[54] A. Farhadi, S. Gundlapalli, M. Shaikh et al., "Susceptibility to gut leakiness: a possible mechanism for endotoxaemia in nonalcoholic steatohepatitis," Liver International, vol. 28, no. 7, pp. 1026-1033, 2008.

[55] N. M. Davies, "Toxicity of nonsteroidal anti-inflammatory drugs in the large intestine," Diseases of the Colon and Rectum, vol. 38, no. 12, pp. 1311-1321, 1995.

[56] J. Eaden, "Review article: the data supporting a role for aminosalicylates in the chemoprevention of colorectal cancer in patients with inflammatory bowel disease," Alimentary Pharmacology and Therapeutics, vol. 18, no. 2, pp. 15-21, 2003.

[57] S. N. Agoff, T. A. Brentnall, D. A. Crispin et al., "The role of cyclooxygenase 2 in ulcerative colitis-associated neoplasia," American Journal of Pathology, vol. 157, no. 3, pp. 737-745, 2000.

[58] D. S. Pardi, E. V. Loftus, W. K. Kremers, J. Keach, and K. D. Lindor, "Ursodeoxycholic acid as a chemopreventive agent in patients with ulcerative colitis and primary sclerosing cholangitis," Gastroenterology, vol. 124, no. 4, pp. 889-893, 2003.
[59] I. A. Mouzas, E. Papavassiliou, and I. Koutroubakis, "Chemoprevention of colorectal cancer in inflammatory bowel disease? A potential role for folate," Italian Journal of Gastroenterology and Hepatology, vol. 30, no. 4, pp. 421-425, 1998.

[60] J. Carrier, A. Medline, K. J. Sohn et al., "Effects of dietary folate on ulcerative colitis-associated colorectal carcinogenesis in the interleukin 2- and $\beta$-microglobulin-deficient mice," Cancer Epidemiology Biomarkers and Prevention, vol. 12, no. 11, pp. 1262-1267, 2003.

[61] B. A. Lashner, P. A. Heidenreich, G. L. Su, S. V. Kane, and S. B. Hanauer, "Effect of folate supplementation on the incidence of dysplasia and cancer in chronic ulcerative colitis. A casecontrol study," Gastroenterology, vol. 97, no. 2, pp. 255-259, 1989.

[62] B. A. Lashner, K. S. Provencher, D. L. Seidner, A. Knesebeck, and A. Brzezinski, "The effect of folic acid supplementation on the risk for cancer or dysplasia in ulcerative colitis," Gastroenterology, vol. 112, no. 1, pp. 29-32, 1997.

[63] S. Matula, V. Croog, S. Itzkowitz et al., "Chemoprevention of colorectal neoplasia in ulcerative colitis: the effect of 6mercaptopurine," Clinical Gastroenterology and Hepatology, vol. 3, no. 10, pp. 1015-1021, 2005. 


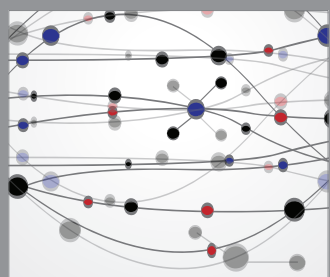

The Scientific World Journal
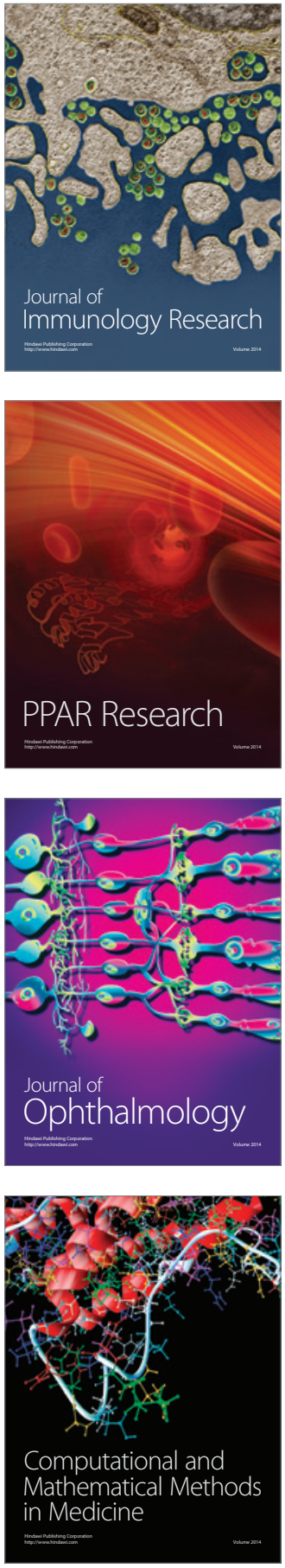

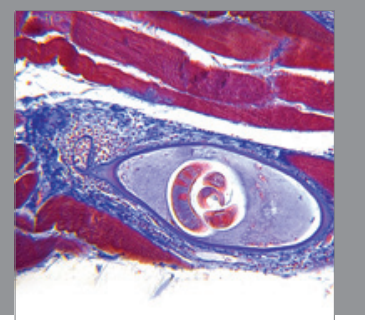

Gastroenterology

Research and Practice
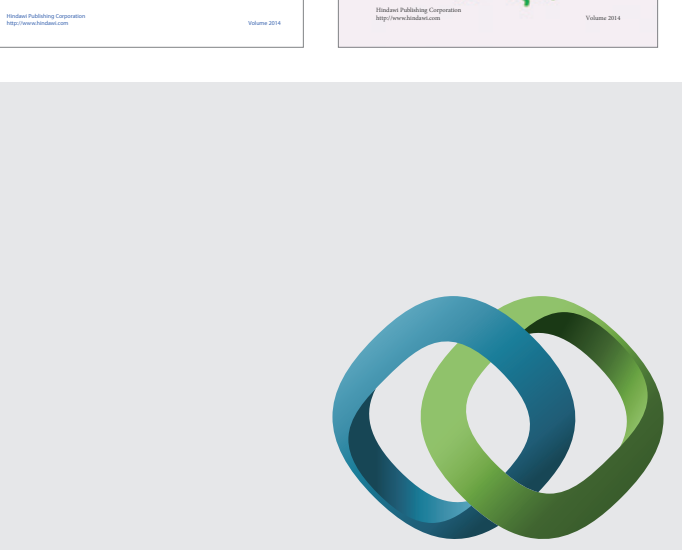

\section{Hindawi}

Submit your manuscripts at

http://www.hindawi.com
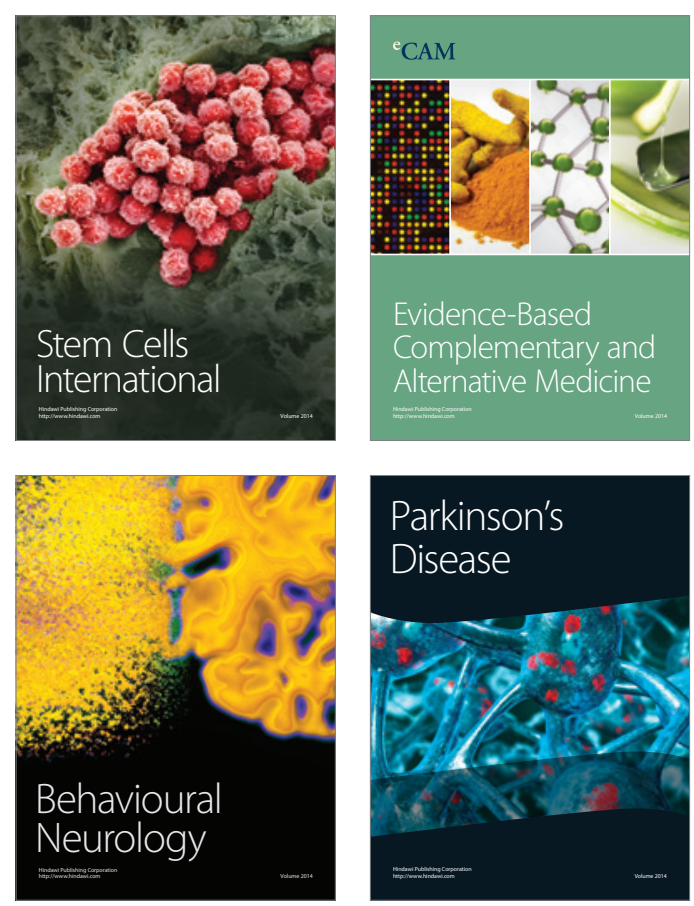

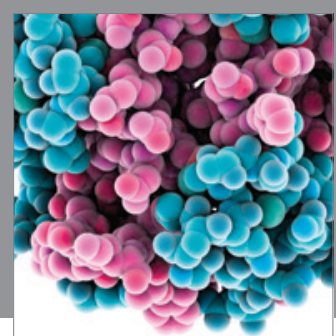

Journal of
Diabetes Research

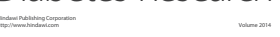

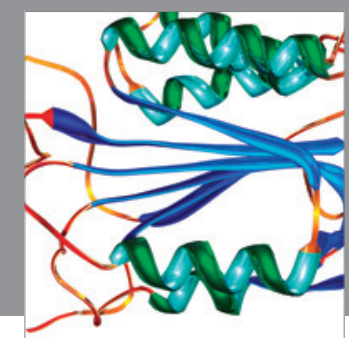

Disease Markers
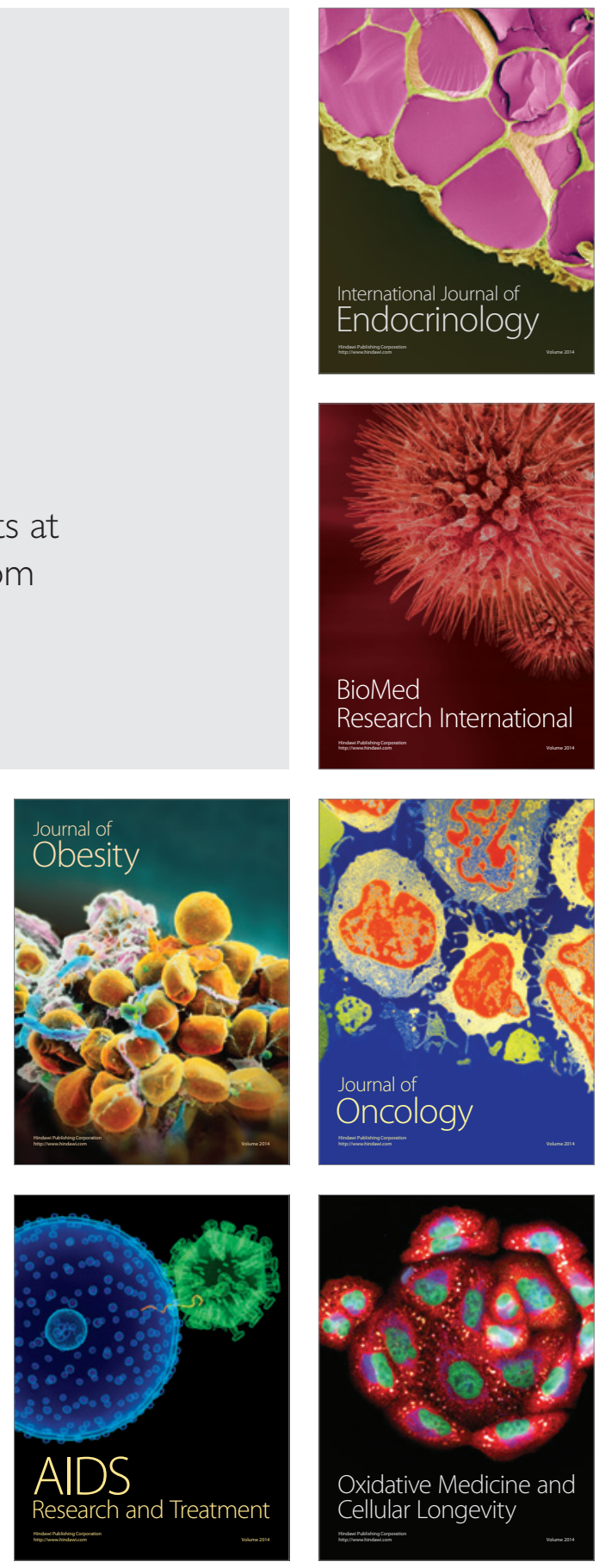